\title{
Formulación de helados a base de pulpa de tamarindo (Tamarindus indica L.) con hidrocoloides y enriquecidos con vitamina C
}

\author{
Formulation of ice cream based on pulp of tamarind \\ (Tamarindus indica L.) with hydrocoloids and enriched \\ with vitamin $C$
}

(iD) Juan Carlos Bravo Aranibar ${ }^{1} \square_{y}$ (iD Noemí Bravo Aranibar ${ }^{2}$

'Universidad Le Cordon Bleu. Lima, Perú

¿Universidad Nacional Mayor de San Marcos. Lima, Perú

\begin{tabular}{|c|c|c|c|}
\hline Recibido: 25/01/2019 & Revisado: 02/04/2019 & Aceptado: 28/05/2019 & Publicado: 30/06/2019 \\
\hline
\end{tabular}

\section{RESUMEN}

Esta investigación se basa en la necesidad de desarrollar una nueva línea de producción de helados de fruta. Actualmente el tamarindo en el mercado nacional se puede encontrar en productos como en marcianos, cremoladas, ingrediente en la cocina, jaleas o como fruto. La propuesta del trabajo es innovadora, ya que se plantea la posibilidad de aprovechar la pulpa del fruto en helados como insumo industrial, debido a la alta concentración de ácido tartárico que se encuentra en la pulpa.

Para el helado se utiliza hidrocoloides (goma guar y goma xantana) para brindarle una textura típica de los helados de fruta con leche, además de ser enriquecido con ácido ascórbico como fuente de vitamina $\mathrm{C}$. Se realizó tres formulaciones de helados que en sus últimas etapas de proceso de mezclado y congelado fueron maquilados en una planta industrial de helados artesanales para tener mejores características sensoriales.

Los helados fueron degustados y evaluados por medio de una encuesta para determinar el nivel de aceptación por medio de preguntas con medición de escala hedónica en relación con color, sabor, textura y apariencia general.

En la percepción de los encuestados se observó la reacción de la sensación del nuevo sabor del ácido tartárico del tamarindo que, en combinación con el ácido ascórbico, ácido láctico de la leche resaltaba aún más la acidez del helado y los hidrocoloides utilizados que les proporcionaron la textura típica de un helado.

Finalmente, el uso en diferentes proporciones de las gomas guar y xantana determinó que la tercera formulación con código 190 obtuvo el mayor nivel de aceptabilidad.

Palabras clave: Tamarindo, formulación, hidrocoloide, ácido tartárico, ácido ascórbico y ácido láctico. 


\section{ABSTRACT}

This research is based on the need to develop a new production line for fruit ice cream. Currently, tamarind in the national market can be found in products such as marcianos, cremoladas, ingredients in the kitchen, jellies or as fruit.

The proposal of the work is innovative, since it raises the possibility of taking advantage of the pulp of the fruit in ice cream as an industrial input, due to the high concentration of tartaric acid found in the pulp. For ice cream, hydrocolloids (guar gum and xanthan gum) are used to provide a typical texture of fruit ice cream with milk, in addition to being enriched with ascorbic acid as a source of vitamin $\mathrm{C}$.

Three ice cream formulations were made that in its last stage mixing and freezing processes were assembled at an industrial artisanal ice cream plant to have better sensory characteristics. Ice creams were tasted and evaluated by means of a survey to determine the level of acceptance by means of questions with hedonic scale measurement in relation to color, flavor, texture and general appearance.

In the perception of the respondents, the reaction of the sensation of the new flavor of tartaric acid of tamarind was observed, which, in combination with ascorbic acid, lactic acid of milk further highlighted the acidity of the ice cream and the hydrocolloids used that gave them the Typical texture of an ice cream. Finally, the use of in different proportions of guar and xanthan gums determined that the third formulation with code 190 obtained the highest level of acceptability.

Keywords: Tamarind, formulation, hydrocolloid, tartaric acid, ascorbic acid and lactic acid.

\section{INTRODUCCIÓN}

El helado es considerado un alimento con mucha aceptación por todos los grupos de edad en el mundo.

Es un producto de alto contenido calórico, debido a la cantidad de carbohidratos que utiliza para su elaboración, según sea el caso. Actualmente se han diseñado formulaciones de helados con usos diferentes de gelificantes como el caso de la goma guar, goma xantan, carboxil metil celulosa, etc.

Sin embargo, los productos que se han elaborado son a base de otras frutas - saborizantes de fresa, maracuyá, piña y otros. No obstante, aún no se ha desarrollado algún producto cuya base sea la fruta de tamarindo. Esto define que no se cuenta una formulación para realizar helados de tamarindo. A este producto en la formulación se le enriquecerá con vitamina $C$ al que se le incorpora ácido ascórbico de grado alimentario de $100 \%$ de pureza, lo que incorpora niveles superiores a los encontrados en forma natural en la fruta de tamarindo.

El alimento enriquecido es la serie de productos alimenticios a los que se le ha incorporado e incrementado el componente del ácido ascórbico.

\section{Características del Fruto}

Barangano, M. (1980) describe al tamarindo con las siguientes características:

Nombre común: Tamarindo

Especie: Tamarindus indica L. 
Familia: Leguminosae

Subfamilia: Caesalpinaide

El tamarindo es un fruto de sabor ácido y dulce cuando está maduro. El fruto en Perú proviene de la zona norte de Chiclayo y Piura. Nuestra industria nacional lo podría utilizar como un excelente ingrediente para preparar helados, jaleas, mermeladas.

En el caso de la gastronomía peruana lo podríamos utilizar en guisos, especialmente para preparar aderezos y salsas, así como en postres, panes, galletas y otros.

Los beneficios nutricionales del tamarindo son debido a que contiene un alto porcentaje de ácido tartárico, presenta minerales como magnesio, calcio, fósforo, azufre, también contiene vitaminas y azúcares saludables que aportan energía y tiene un alto porcentaje de contenido de fibra.

Escudero, J. (2008) indica que el tamarindo destaca por ser una de las frutas que concentra mayor cantidad de calcio, aproximadamente $74 \mathrm{mg}$, alto valor de hierro $2,8 \mathrm{mg}$ y potasio $628 \mathrm{mg}$ de concentración por $100 \mathrm{~g}$ de pulpa. Además, tiene propiedades benéficas para la salud como ser usado como laxante para ayudar a evitar el estreñimiento por el contenido, principalmente, de ácido tartárico.

\section{Helados}

Para soluciones prácticas, los helados son productos alimenticios que se llevan a estado sólido o pastoso por medio de la congelación.

Hernández, M. (2014) refiere que de acuerdo con Mataix J, Solanes E. y Rodriguez E. define que el helado es un alimento congelado que se somete a un proceso de agitación, saborización, mezcla de estabilizantes, edulcorantes o azucares para darle cuerpo al mismo.

Este es el resultado de una mezcla homogénea, pasteurizada, que dependiendo del producto final que se quiera obtener tendrá determinada cantidad de aire, porcentaje de fruta, agua, crema, grasa, azúcar, estabilizante, etc.

\section{Hidrocoloides utilizados en helados}

Ordoñez, J. (1998) menciona que bajo la denominación de gomas se incluye un amplio grupo de polisacáridos solubles en agua procedentes de vegetales terrestres o marinos o de origen microbiano y que poseen la capacidad de aumentar la viscosidad de una solución y de formar geles debido a su carácter altamente hidrófilo. Las gomas son muy utilizadas en la tecnología alimentaria como gelificantes y espesantes.

\section{Goma xantana}

Para poder emulsificar el helado, previamente se evaluó la ficha técnica de la empresa Xinjiang Meihua Amino Acid Co. Ltd. que describe las especificaciones técnicas de la goma xantana.

Dentro de estos parámetros técnicos las características de esta goma extracelular de ácido heteropolisacarido que es producido por la fermentación de bacterias Xanthomonas campestris, hecho dealmidón y otro carbohidrato a través de procesos de cultivos, extracción, evaporación y molienda, esto es ampliamente usado en industrias de alimentos, aceites, minas, farmacia y textiles.

Tiene propiedades reológicas excepciona- 
les, es muy efectiva como estabilizante de sistemas basados en agua y tiene numerosas aplicaciones en la industria de alimentos entre las cuales se encuentran: salsas emulsionadas, productos lácteos, productos bajos en calorías (Taherian et. al., 2007).

Es muy utilizada en la industria alimentaria debido a las siguientes características: solubilidad en agua fría o caliente; alta viscosidad a bajas concentraciones; ausencia de cambios discernibles en la viscosidad de sus soluciones en el intervalo de $0-100^{\circ} \mathrm{C}$, lo que la hace única entre los hidrocoloides: solubilidad y estabilidad en sistemas ácidos; excelente compatibilidad con la sal; interacción con otros hidrocoloides; capacidad de estabilizar suspensiones y emulsiones; y buena estabilidad de las soluciones frente a la congelación / descongelación.

Es ideal para estabilizar dispersiones, suspensiones y emulsiones acuosas.

Esta goma está compuesta de fibra que es un polisacárido por lo cual no se metaboliza en el tubo digestivo, por ello es eliminado por las heces.

\section{Goma guar}

Para la textura del helado no solo es necesario el uso de una goma, es necesario incluir el uso de otro gelificante, se revisó la ficha técnica de la empresa Shree Vijaylaxmi Enterprises que describe las especificaciones técnicas de la goma guar de ser un polvo de alta pureza que es obtenido del endospermo de la semilla de la planta guar Cyamopsis tetragonoplus.

La goma guar es de peso molecular alto, que tiene de base manosa y galactosa.

El promedio de proporción de estos azucares es aproximadamente de 2:1. La goma guar es un vegetal natural que no requiere calor para desarrollar viscosidad alta.

Los beneficios y características son: soluble en agua fría, alta viscosidad, hidratación rápida y estabilidad en congelación y descongelación.

Las aplicaciones de la goma guar es en los siguientes alimentos: helados, tallarines, caramelos, sopas instantáneas, salsas y otros.

Mora, F. (2013) describe que la goma guar se encuentra en las semillas de la planta Cyamopsis tretragonalobus (originaria de India y cultivada en los Estados Unidos).

Este aditivo alimentario se utiliza para producir soluciones altamente viscosas que se pueden hidratar en agua fría. Las sales minerales no afectan su hidratación.

Estehidrocoloidesedisuelvecompletamente en agua fría, produce soluciones no newtonianas de comportamiento pseudoplásticos. Esta goma no gelifica otorga cuerpo al producto.

\section{Ácido ascórbico}

La vitamina $C$ en el reino vegetal del Perú se encuentra en muchas frutas y verduras. Se tiene una fruta con una concentración de vitamina $C$ de manera excepcional como es el caso del camu camu que tiene aproximadamente de 2000 - 2780 mg de vitamina C. En verduras como el perejil alcanza concentración de 130 mg de vitamina C. 
Kimball, D. (1999) indica que, para los ácidos, otro componente fundamental de los zumos de cítricos y las bebidas a base de zumo de cítricos, proporcionan el característico gusto estimulante de los cítricos.

Como el ion hidronio es el que imparte este gusto, se puede utilizar la mayoría de los ácidos. Sin embargo, no todos los ácidos están autorizados para uso alimentario.

\section{Viscosidad}

La densidad es la relación entre la masa y volumen. Quiere decir a mayor masa la densidad es mayor.

Muchos autores refieren que un kilo de algodón de azúcar es mucho menos denso que un kilo de carne, pues el primero ocupa mucho más volumen. La leche es más densa que el aceite. El único que tiene la masa y el volumen igual es el agua cuya densidad es de $1.000 \mathrm{~g} / \mathrm{cm}^{3}$.

\section{Overrun}

Hernández, M. (2014) define que el término "overrun" se refiere al rendimiento o aumento de volumen al batido. Se define como el índice de aireación o cantidad de aire agregado a la mezcla en porcentaje sobre la misma en volumen.

Se cita a Saturnino, M. quien comenta que la introducción de aire al sistema provoca que se obtenga un producto trifásico (sólido, líquido y gas).

Este aire no interviene de manera importante en la transferencia de calor sobre la pared, pero si puede intervenir en una distribución del tamaño de cristales.

\section{Población de estudio}

El trabajo de investigación realizado en Lima determina que una definición adecuada de población debe realizarse a partir de los siguientes términos: alcance, tiempo, elementos de muestreo.

La pregunta por realizar para conocer el tamaño de la muestra es de cuántos alumnos tendría que estudiar de una población de 114 alumnos desde el segundo al quinto ciclo de turismo y hotelería y gastronomía del instituto Latino para conocer la evaluación sensorial de la prueba de aceptabilidad de las diferentes formulaciones de helado a base de pulpa de tamarindo con hidrocoloides y enriquecido con vitamina $\mathrm{C}$.

La seguridad $=95 \%$; precisión = $6 \%$; proporción esperada = asumamos que puede ser próxima al 50 \% de éxito y $50 \%$ de fracaso. Por tanto, la muestra es:

$$
\mathrm{n}=\frac{114 * 1.96^{2 *} 0.50 * 0.50}{(0.06)^{2}(114-1)+1.96^{2 *} 0.5 * 0.50}=80
$$

Los alumnos degustaron las tres formulaciones de helados quienes determinaron mediante una encuesta (escala hedónica) que helado fue el mayor en nivel de aceptabilidad.

Debemos tener en cuenta que la degustación de los helados se realizó en hora promedio de las once de la mañana. Hora propicia después de un tiempo alejado desde que ellos hayan ingerido su desayuno.

La observación cualitativa de parte de nosotros fue evidente en evidenciar las reacciones diferentes al nuevo sabor de ácido tartárico del tamarindo. Algunos de los encuestados preguntaban por el sabor nuevo de sus características. 


\section{MATERIALES Y MÉTODOS}

Formulaciones realizadas de los helados de tamarindo

En la Tabla 1 se presentan las tres formulaciones de los helados a base de pulpa de tamarindo con hidrocoloides y enriquecidos con vitamina $\mathrm{C}$.

Previamente se realizaron varias formula- ciones para evaluar la percepción del sabor de las diferentes preraciones del helado en relación a la concentración de la pulpa de tamarindo debido a que el sabor característico de la fruta se debe por contener el ácido orgánico que es el tartárico que otorga una sensación en la boca de un sabor agridulce que se intensifica con la mezcla de otros ácidos orgánicos.

Tabla 1. Penas impuestas por la comisión de actos de corrupción

\begin{tabular}{lccc}
\hline Número de formulación & $\begin{array}{c}\text { Fórmula } \mathbf{1} \\
\text { Código } \mathbf{1 5 0}\end{array}$ & $\begin{array}{c}\text { Fórmula 2 } \\
\text { Código 170 } \\
\text { Porcentaje }\end{array}$ & $\begin{array}{c}\text { Fórmula 3 } \\
\text { Código 190 }\end{array}$ \\
\hline Pulpa de tamarindo & 26,43 & 26.43 & 26.43 \\
Agua & 29.23 & 29.23 & 29.23 \\
Leche evaporada & 23.56 & 23.56 & 23.56 \\
Azúcar rubia & 19.16 & 19.16 & 19.16 \\
Lecitina de soya & 0.88 & 0.88 & 0.88 \\
Goma xantana & 0.44 & 0.00 & 0.22 \\
Goma guar & 0.00 & 0.44 & 0.22 \\
Ácido ascórbico & 0.30 & 0.30 & 0.30 \\
Total & $100 \%$ & $100 \%$ & $100 \%$ \\
\hline
\end{tabular}

Procedimiento de elaboración de los helados de tamarindo

1. Se contactó al proveedor de pulpa de tamarino para que enviara las muestras de pulpa de tamarindo, proveniente la fruta de Piura. Fue adquirido en el Mercado Mayorista de Frutas de la Victoria.

El proveedor de la fruta de tamarindo dejó remojando 12 horas en agua cuya temperatura inicial es de $100{ }^{\circ} \mathrm{C}$ y progresivamente decrece con el fin de extraer la pulpa. Luego del remojado la pulpa es dispuesta en una pulpeadora ubicado en la universidad La Unión.

2. Se compraron los ingredientes principales: goma guar, goma xantana, ácido ascórbico y lecitina de soya de la empresa Aromas del Perú.

La leche evaporada y el azúcar rubia se 
adquirieron en una tienda comercial.

3. Se procedió a revisar las fórmulas originales del helado de tamarindo, que se hicieron previamente dos formulaciones anteriores para realizar la dosificación final por medio de una balanza analítica y tener la precisión de los pesos respectivos de acuerdo con el porcentaje correspondiente que totaliza el $100 \%$.

4. La base del helado se preparó un día antes de ser mezclado con la pulpa de tamarindo. La base consiste en la mezcla inicial de los sólidos como el azúcar, goma xantana, goma guar, lecitina de soya, posteriormente es unida con los líquidos a temperatura de aproximadamente $70^{\circ} \mathrm{C}$.

5. La base se sometió a batido para poder crear el overrun, el cual le confiere la consistencia y característica de helado de una textura agradable.

Luego se procedió a una pasteurización de temperatura a $70{ }^{\circ} \mathrm{C}$ por un tiempo de 30 minutos.

6. La base fue enfriada y se vertió en jarras con tapa para realizar la maduración del base mínimo se dejó en reposar por 6 horas de acuerdo a las técnicas aprendidas en la elaboración de helados por colaboradores de teorías de libros y tesis.

7. La base y la pulpa son transportadas hacia la empresa de helados donde se mezclan en una licuadora industrial por un tiempo de 30 segundos.

8. Seguidamente se agregó a la mezcla en una mantecadora industrial a una temperatura de -7 por un tiempo de 10 minutos.

9. El helado se envasó en moldes de 6 litros por cada tipo de formulación. Posteriormente los helados de cada formulación son almacenados en una congeladora a una temperatura de $-18^{\circ} \mathrm{C}$.

10. Se cogió una muestra de $500 \mathrm{~g}$ de helado para ser llevado al laboratorio para su evaluación microbiológica y fisicoquímica.

11. El helado una vez congelado se envasó y se vertió en envases pequeños de $10 \mathrm{ml}$ de acuerdo al tipo de formulación.

12. Los helados envasados en los pequeños recipientes son transportados al instituto Latino donde se realizó las pruebas de preferencia de análisis sensorial de una muestra de 80 alumnos.

\section{Prueba microbiológica y fisicoquímica}

Los ensayos microbiológicos y fisicoquímicos del helado de tamarindo se realizaron en el Laboratorio Certificaciones Alimentarias Hidrobiológicas y Medioambientales SAC (CAHM).

El producto se entregó en congelación. La fecha de ejecución de ensayos del 25/01/2019 al 30/01/2019.

\section{RESULTADOS}

Las pruebas microbiológicas se realizaron en base al análisis de recuento de microorganismos aerobios, coliformes, Staphylococcus aureus, Listeria monocytogenes y Salmonella.

Con estos resultados se accedió a la degustación de los productos. Estos datos se pueden observar en la tabla 2. 
Tabla 2. Ensayos microbiológicos del helado de tamarindo

\begin{tabular}{lccc}
\hline ITEM & Parámetros & Unidades & Resultados \\
\hline 01 & Recuento de microorganismos aerobios & UFC/g & $<10^{(4)}$ \\
02 & Enumeración de coliformes & UFC/g & $<3$ \\
03 & Recuento de Staphylococcus aureus & UCF/g & $<10^{(4)}$ \\
04 & Detección de Listeria monocytogenes & Listeria & \\
& & monocytogenes $/ 25 \mathrm{~g}$ & Ausencia \\
05 & Detección de Salmonella & Salmonella/25 g & Ausencia \\
\hline
\end{tabular}

Fuente: Laboratorio CAHM - Enero 2019

En relación con el análisis fisicoquímico, en la tabla 3, se describe la acidez del producto expresada en acido tartárico.

Los grados brix que se muestran son altos, esto responde al sabor dulce del helado y el pH estabilizado a 3.5, garantiza que el producto no podrá desarrollar bacterias.

En las normas alimentarias peruanas indica que el uso para proteger el alimento por acidez debe ser el pH de 4.6 como máximo.

Tabla 3. Acidez del helado de tamarindo

\begin{tabular}{lccc}
\hline ITEM & Parámetros & Unidades & Resultados \\
\hline 01 & $\begin{array}{c}\text { Acidez (expresada en } \\
\text { ácido tartárico) }\end{array}$ & $\%$ & 0,52 \\
02 & Grados Brix & ${ }^{\circ}$ Brix & 32 \\
03 & $\mathrm{pH}$ & Unidad del 1-14 & 3,5 \\
\hline
\end{tabular}

Fuente: Laboratorio CAHM - Enero 2019

Tabulación y análisis de la prueba de aceptación

Pregunta $\mathrm{N}^{\circ} 1$

Según su evaluación, ¿Cómo calificaría usted el color del helado de tamarindo de la formulación con código 190? 


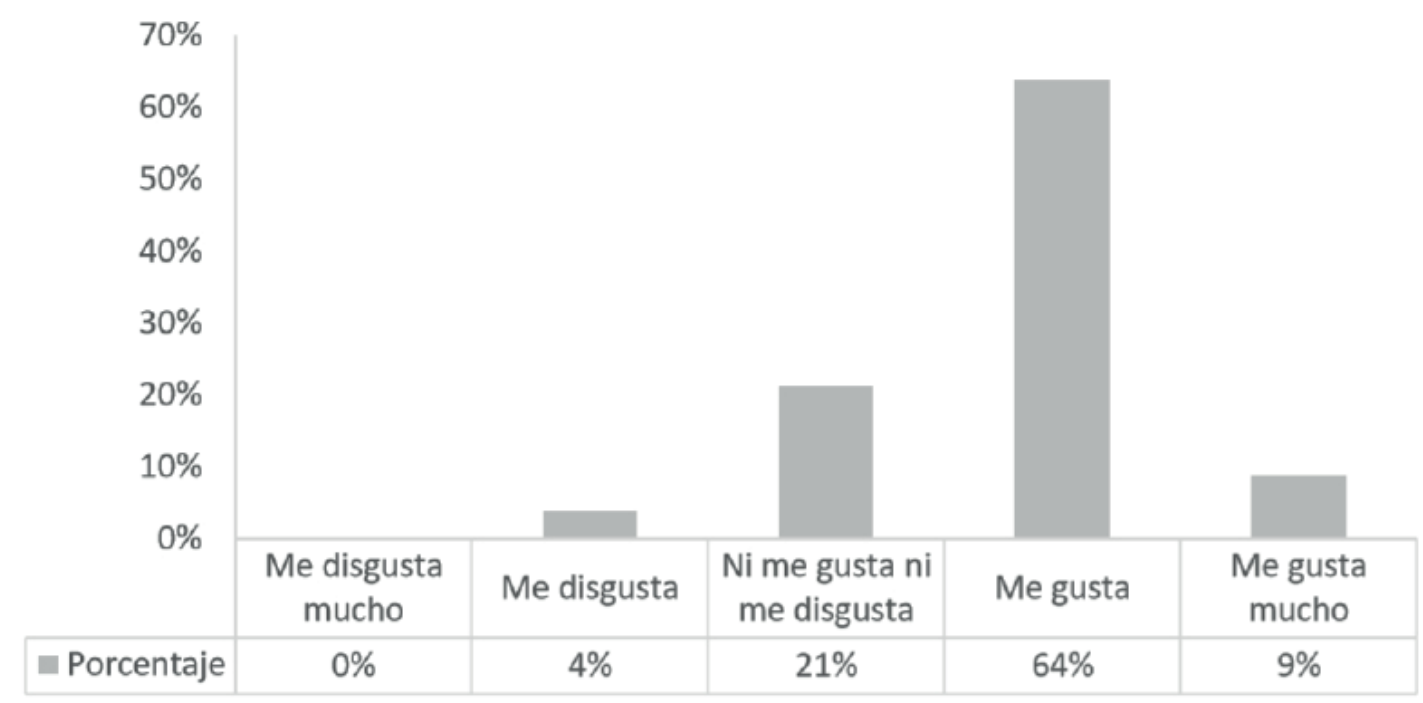

Figura 1. Color de helado de tamarindo 190.

En la figura 1 se observa, que el $9 \%$ les gusta mucho el color del helado de código 190, 64 \% de personas les gusta, $21 \%$ respondieron de ni me gusta ni me disgusta, mientras que al 4 $\%$ de las personas les disgusta.

\section{Pregunta $\mathrm{N}^{\circ} 2$}

Según su evaluación, ¿cómo calificaría usted el helado de tamarindo respecto a la textura? Para ello, se realizó el análisis sensorial respectivo para las tres formulaciones elaboradas en el proyecto de investigación.

En la figura 2 se observa los resultados de la textura del helado 190.

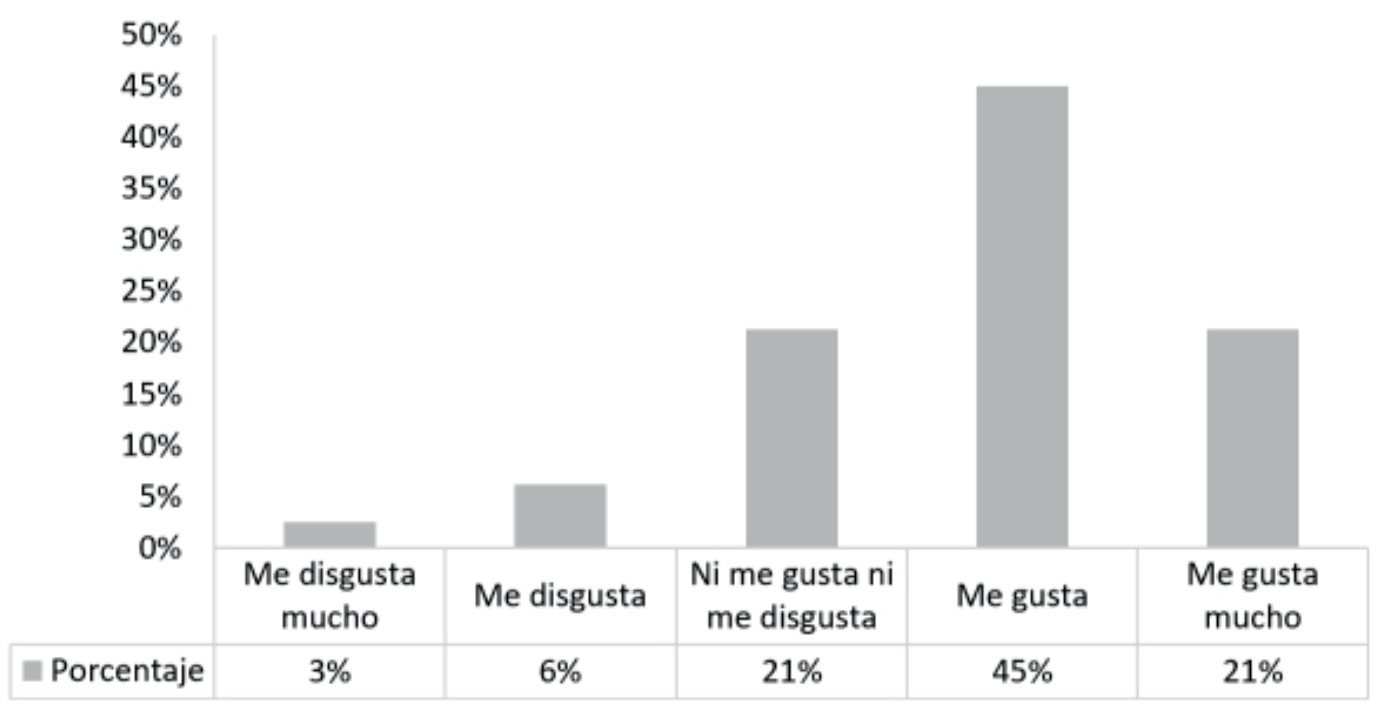

Figura 2. Textura del helado de tamarindo 190. 
En la fig. 2 se puede observar que el $21 \%$ les gusta mucho la textura del helado código 190, $45 \%$ de personas les gusta, $21 \%$ respondieron de ni me gusta ni me disgusta, mientras que al $6 \%$ de las personas les disgusta.
Así como al 3 \% le disgusta mucho.

\section{Pregunta $\mathrm{N}^{\circ} 3$}

Según su evaluación, ¿cómo calificaría usted el sabor del helado de tamarindo 190?

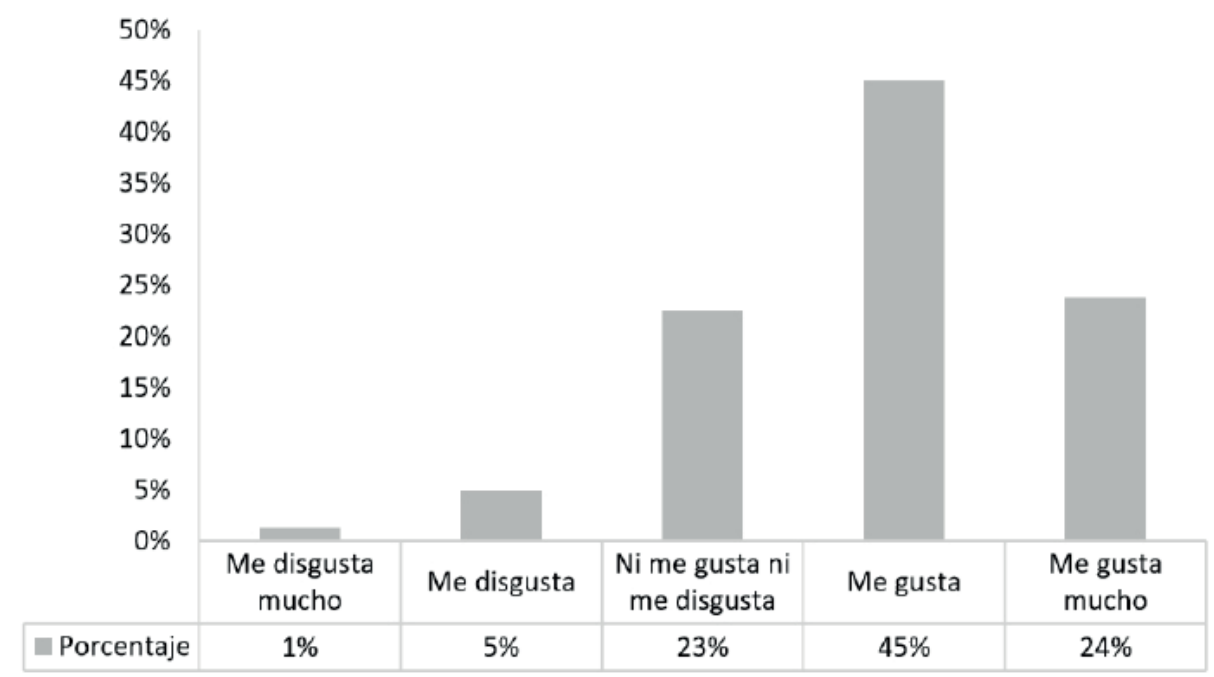

Figura 3. Sabor del helado de tamarindo 190.

La fig. 3, muestra que el $24 \%$ les gusta mucho el sabor del helado código 190, 45 \% de personas les gusta, $23 \%$ respondieron de ni me gusta ni me disgusta, mientras que al $5 \%$ de las personas les disgusta. Así como el $1 \%$ le disgusta mucho.

\section{Pregunta $\mathrm{N}^{\circ} 4$}

Según su evaluación ¿cómo calificaría usted la aceptabilidad general del helado?

En esta última pregunta se describe los resultados de las tres formulaciones de helado. En primer lugar iniciamos con el helado 150.

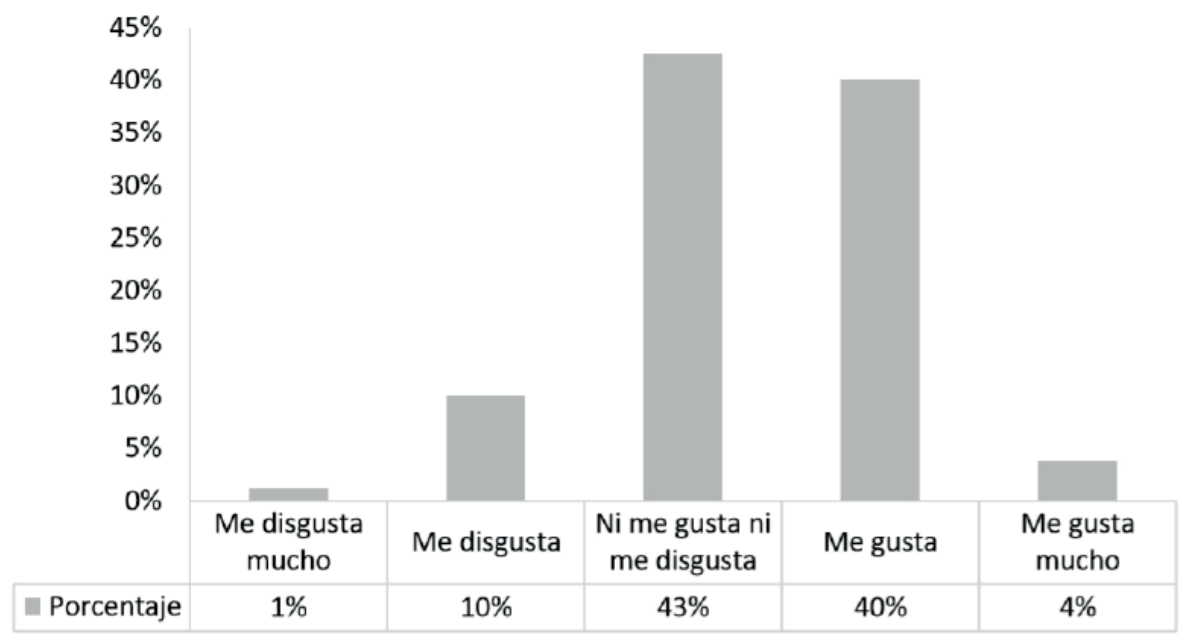

Figura 4. Aceptabilidad general del helado de tamarindo 150. 
En la fig. 4 se puede observar que el $4 \%$ de personas les gusta mucho la aceptabilidad general del helado código 150, el $40 \%$ respondieron que les gusta, mientras el $43 \%$ respondió que el helado ni me gusta ni me disgusta. El $10 \%$ de las personas el helado les disgusta y al $1 \%$ le disgusta mucho.

La aceptabilidad general de la segunda formulación del helado de tamarindo código 170 referente a sus resultados de la encuesta se describen en la fig. 5 .

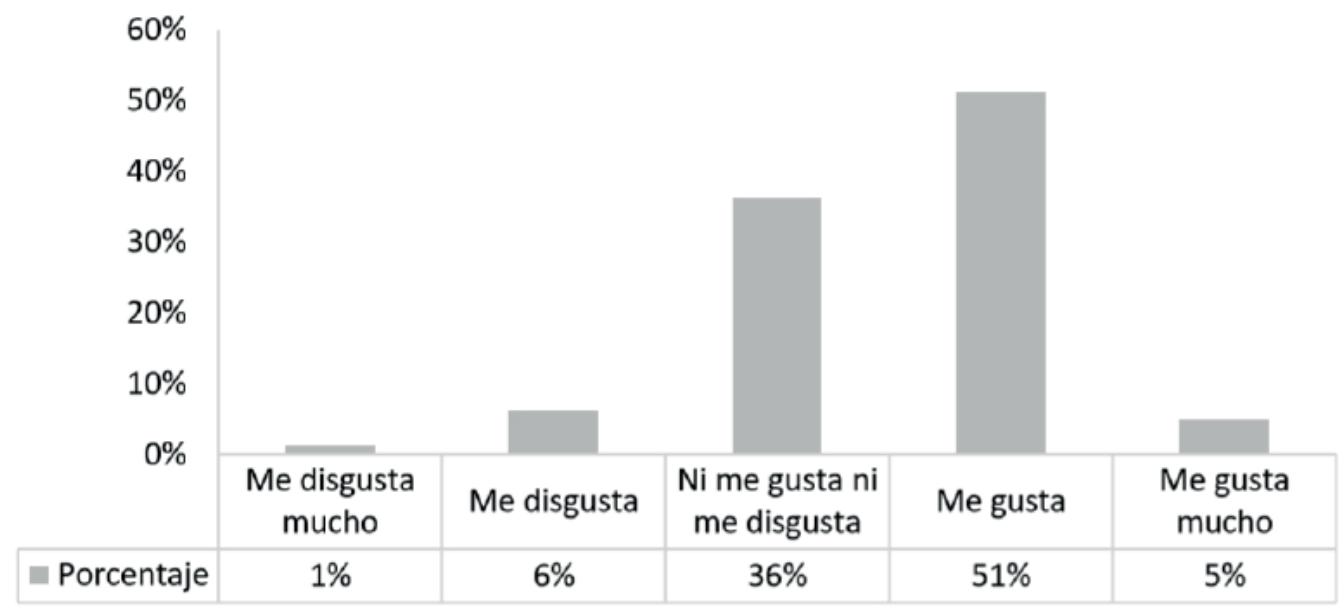

Figura 5. Aceptabilidad general del helado de tamarindo 170.

Se describe en la fig. 5 que la aceptabilidad general del helado de la segunda formulación código 170 es de 5 \% que les gusta mucho, $51 \%$ de personas les gusta, $36 \%$ respondieron de ni me gusta ni me disgusta mientras que al $6 \%$ de las personas les disgusta. Así como al $1 \%$ le disgusta mucho.

A continuación, se indica en la fig. 6 el nivel de aceptabilidad general del helado de tamarindo de la tercera formulación código 190.

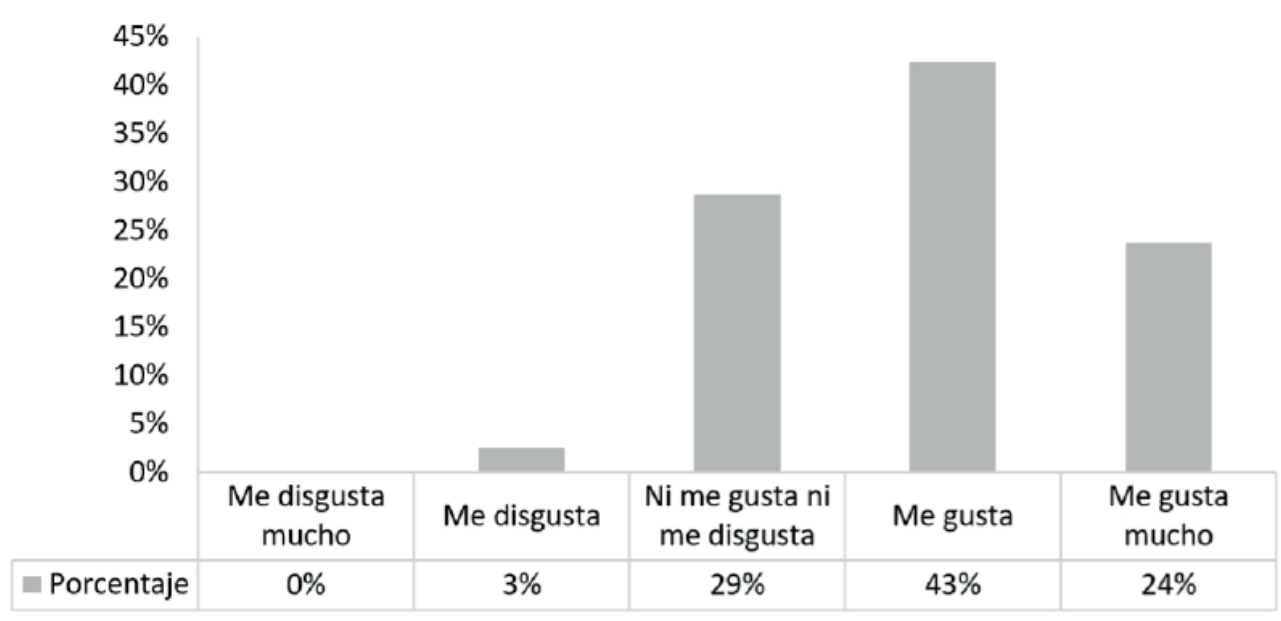

Figura 6. Aceptabilidad general de helado de tamarindo 190. 
La fig. 6 muestra que el $24 \%$ les gusta mucho la aceptabilidad general del helado de la tercera formulación código 190, 43 \% de personas les gusta, $29 \%$ respondieron de ni me gusta ni me disgusta, mientras que al $3 \%$ de las personas les disgusta.

Para poder definir que helado ha tenido la más alta preferencia en relación con el nivel de aceptabilidad general. Se realizó la comparación del helado de la tercera formulación código 190 con la primera formulación código 150 y con la segunda formulación código 170.
En la fig. 7 se describe la comparación del nivel de aceptabilidad general del helado con mayor preferencia. La escala hedónica que se utilizó para medir el nivel de aceptabilidad por parte de los consumidores fue adecuada ya que esta escala nos demostró de una manera fácil la formulación de helado que tuvo mayor aceptación.

Las fórmulas en la figura 7 evalúan la aceptabilidad general en comparación al color, sabor y textura de cada helado de tamarindo.

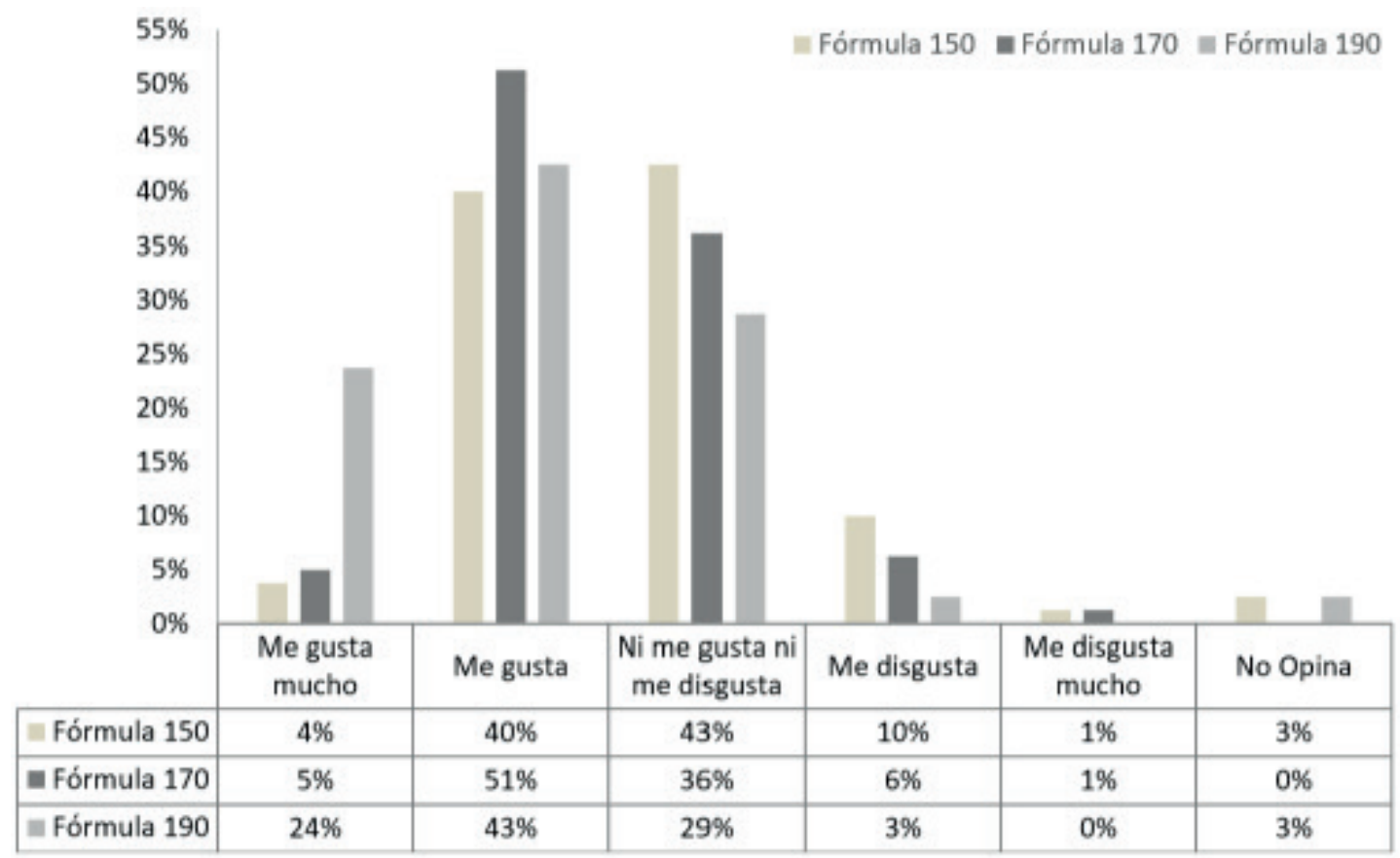

Figura 7. Comparación de aceptabilidad general de fórmulas.

En la práctica la "aceptabilidad general" representa un promedio, que el entrevistado percibe, de las variables color, textura y sabor que evalúa después de probar los tres tipos de helados; en tal sentido, al comparar los resultados en esta pregunta, observamos un cambio favorable en la respuesta "me gusta mucho" hacia la formulación 190 hasta en 20 puntos porcentuales y una reducción en las respuestas negativas hacía esta formulación. Una mayor migración positiva en la fig. 8 se puede observar las preferencias por la tercera formulación de código 190, se observa que del 40,1\% que respondieron "me gusta" por la formulación 150, el 22,5 \% también tiene la misma preferencia por la formulación 190, a un $8,8 \%$ le gusta mucho, un $7,5 \%$ le es indiferente y sólo un 1,3\% no opina. Del $42,7 \%$ de los que eran 
indiferentes a la primera formulación código 150 ahora el $16,3 \%$ mantiene respuesta similar y un $13,8 \%$ mejora su preferencia a "me gusta" e incluso un 11,3 \% hacía "me gusta mucho" y solo un $1,3 \%$ mantiene respuesta similar.
También el 10,1 \% que no les gustaba la formulación 150, mejoró su percepción hacia la formulación 190, con un 1,3 \% "le gusta mucho", 5,0 \% "le gusta" y el resto 3,8 \% se manifiesta indiferente.

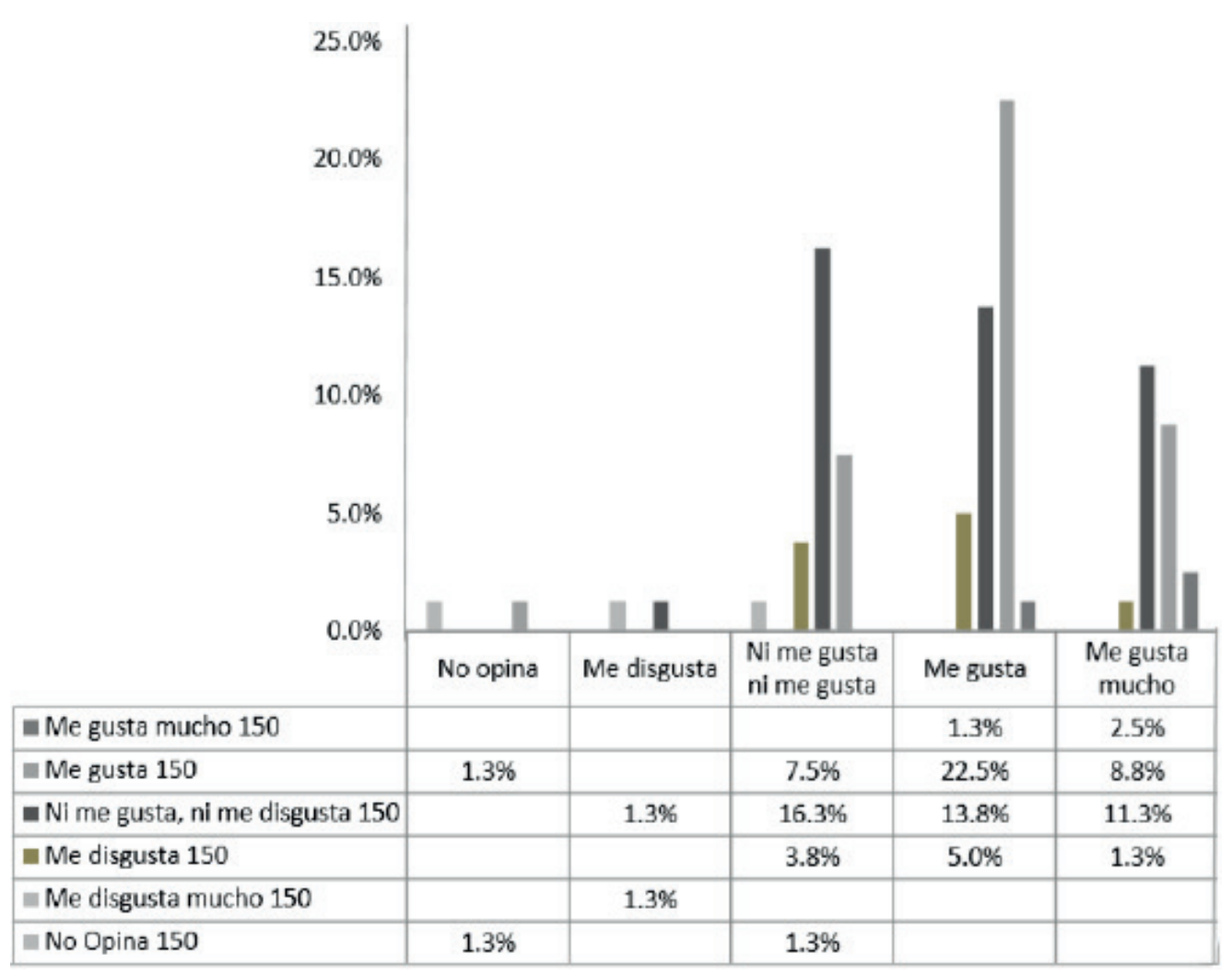

Figura 8. Composición de nivel de aceptabilidad general de helado 190 respecto al helado 150.

Se puede observar que en la fig. 9, la migración positiva a las preferencias por la formulación 190 ha sido mucho mejor, se observa que del $51,3 \%$ que respondieron "me gusta" por la formulación 170, el 22,5 \% también tiene la misma preferencia por la formulación 190, a un $15 \%$ Le gusta mucho, un $10 \%$ le es indiferente, y sólo un 1,3\% no le gusta.

Del $36,3 \%$ de los que eran indiferentes a la formulación 170 ahora el 16,3\% mantiene respuesta similar y un $15 \%$ mejora su preferencia a "me gusta" e incluso un $5 \%$ hacía "me gusta mucho".

También el 6,3 \% que no les gustaba la formulación 170, mejoró su percepción hacia la formulación 190, con un 2,5 \% "le gusta mucho", 2,5 \% "le gusta" y el resto 1,3\% se manifiesta indiferente. 


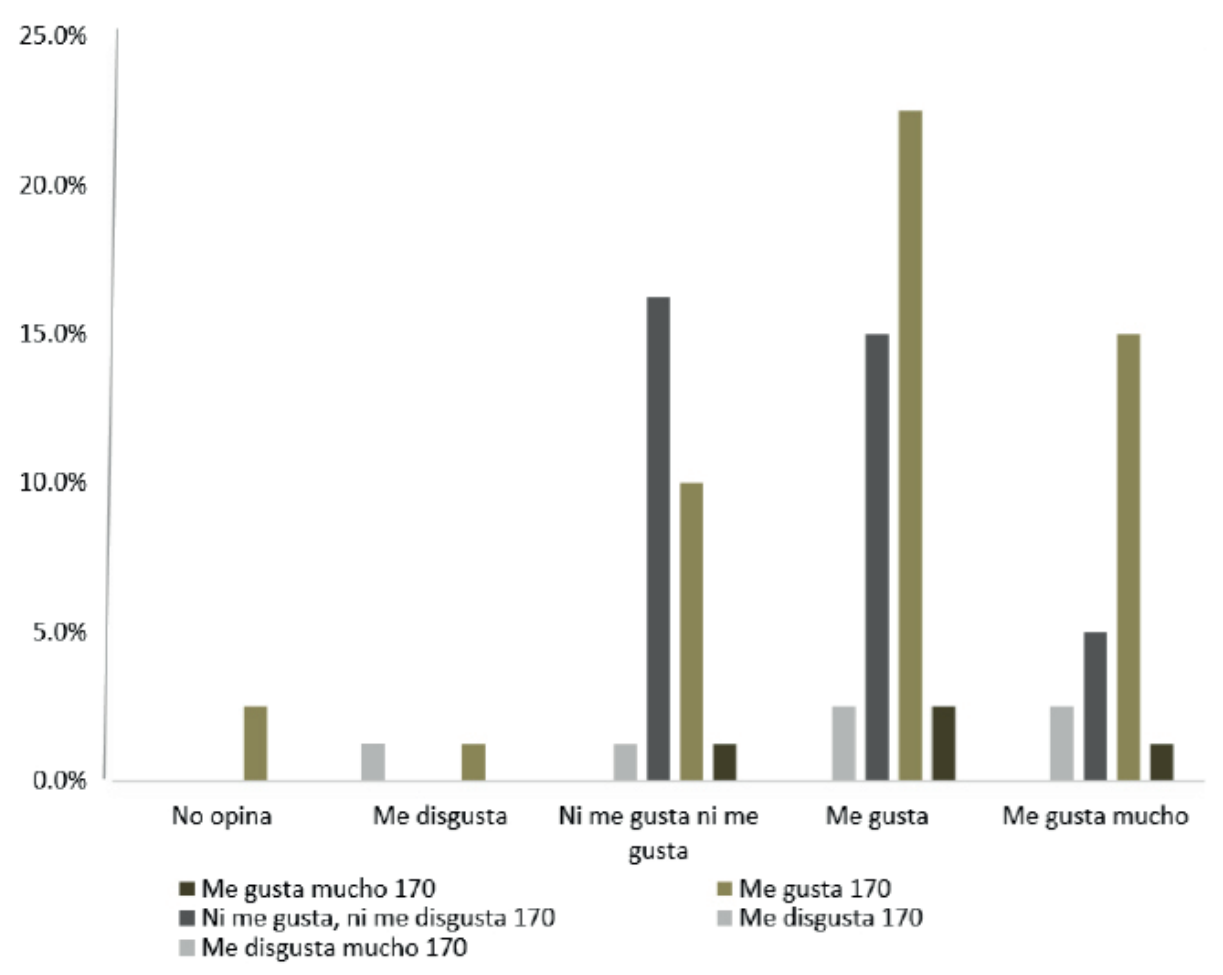

Figura 9. Composición de nivel de aceptabilidad general de helado 190 respecto al 170.

\section{DISCUSIÓN}

De acuerdo con la Resolución Ministerial 5912008/Minsa. Normasanitariaqueestablecelos criterios microbiológicos de calidad sanitaria e inocuidad para los alimentos y bebidas de consumo humano, existen niveles máximos de microorganismos de aerobios, coliformes, Sthaphylococcus aureus, asimismo, define que las muestras de helados deben tener ausencia en una muestra de 25 gramos de helados de Listeria monocytogenes y en una muestra de 25 gramos debe tener también ausencia de Salmonella.

Por tanto, de acuerdo con la tabla 2 el helado de tamarindo cumple con los parámetros de análisis microbiológicos.

Los análisis fisicoquímicos cumplen también los parámetros en el cual la importancia debe estar dada la acidez expresada en ácido tartárico en el producto fue de 0,52\%.

En el proyecto la "aceptabilidad general" representa un promedio, en el cual los entrevistados percibieron y midieron a las variables color, textura y sabor que evaluaron después de probar las tres formulaciones de helados; en tal sentido al comparar los resultados en esta pregunta, observamos un cambio favorable en la respuesta "me gusta mucho" hacia la formulación 190 hasta en 20 puntos porcentuales, principalmente de la percepción del helado de tamarindo y una marcada reducción en las respuestas negativas hacía esta formulación, en comparación con las formulaciones 150 y 170 .

Ávila, F. y Sánchez, J. (2016) en su trabajo las mejores dosificaciones de goma para la 
elaboración del néctar de tamarindo, fueron aquellas que se les incorporó goma Xanthan al $2 \%$ y $3 \%$, esto en relación a la estabilidad y a la aceptación sensorial favorable; sin embargo, la dosificación del $4 \%$ de goma Xanthan presentó una estabilidad mayor a todos los tratamientos porque no permitió la precipitación de los sólidos, pero obtuvo aceptación sensorial desfavorable.

En el caso de nuestro trabajo de helados como máximo se incorporó en combinación de $0.44 \%$ de la goma xantana y guar.

Barandiaran, F. (2013) determinó que con el uso de $0,07 \%$ CMC; $0,25 \%$ goma guar y $0,68 \%$ goma xantana como mezcla óptima para una salsa de alcachofa.

Estos porcentajes no supera las cantidades de hidrocoloides de $0.44 \%$ usados en nuestros helados.

Hernández, M. (2014) para determinar el nivel de agrado de las formulaciones elegidos por los jueces consumidores.

Se utilizaron escalas de intervalo ya queayudoa determinar el grado de satisfacción, preferencia o aceptabilidad de las formulaciones.

En nuestro trabajo el nivel de aceptabilidad de nuestros helados se determinó por medio de una escala hedónica.

El procesamiento de la información respondió a realizar tablas y gráficos, lo cual se efectuó mediante el empleo de hojas de cálculo excel con administración de la base de datos mediante tablas dinámicas, lo que permitió realizar los cruces y correlaciones pertinentes.

Por tanto, la hipótesis planteada en el proyecto que la tercera formulación de la mezcla de goma xantana y goma guar en partes iguales seria la que tendría mayor nivel de preferencia es aceptada de acuerdo con la decisión de los panelistas.

\section{CONCLUSIONES}

Se desarrollaron tres formulaciones de helados a base de pulpa de tamarindo con hidrocoloides y enriquecidos con vitamina C.

En el caso de los hidrocoloides se utilizaron en la primera formulación goma xantana, en la segunda formulación goma guar y la tercera formulación la mezcla de las dos gomas en partes iguales. En el caso del ácido ascórbico se fortificó en la misma cantidad para las tres formulaciones.

De las tres formulaciones se muestreo al azar un helado, el producto cumplió con los requerimientos de análisis microbiológicos y fisicoquímicos de acuerdo con la Resolución Ministerial 591-2008/MINSA que aprueba la norma sanitaria que establece los criterios microbiológicos de calidad sanitaria e inocuidad para los alimentos y bebidas de consumo humano.

El helado de tamarindo que tuvo mayor nivel de aceptabilidad general fue la tercera formulación que fue degustado con el código 190. Esta formulación utilizó la mezcla de la goma xantana y goma guar en partes iguales.

El helado de tamarindo que tuvo menor nivel de aceptabilidad general fue la primera formulación que fue degustado con el código 150. Esta formulación utilizó solamente el hidrocoloide de la goma xantana.

El nivel de aceptabilidad en relación con el sabor en las tres formulaciones fue positivas debido a los sabores proporcionados de la pulpa de tamarindo que le confirió el 
ácido tartárico; además del sabor del ácido ascórbico que se le agregó como ingrediente y el aporte del ácido láctico de la leche evaporada.

\section{REVISIÓN BIBLIOGRÁFICA}

Ávila, F., y Sánchez, J. (2016). Influencia de estabilizantes Goma Guar y Goma Xantana en la calidad Físico - Química y Organoléptica del Néctar de Tamarindo (Tamarindus indica L.). Tesis para Título profesional de Ingeniero Agroindustrial. Escuela Superior Politécnica Agropecuaria de Manabí Manuel Félix López. Calceta. Recuperado de http://repositorio. espam.edu.ec/bitstream/42000/551/1/ TAl108.pdf

Baragano, M. (1980). Technology of clarified tamarind juice. FAO. 2003. Food and Agriculture organization of United Nations. Tablas nutricionales de América Latina (Latin foods 1997).

Barandiaran, F. (2013). Efecto de la concentración de CMC, Goma Guar y Goma Xantana sobre la Sinéresis, características reológicas y consistencia sensorial en Salsa de Alcachofa (Cynara scolymus L.) variedad Imperial Star. Tesis para el Título profesional de Ingeniero en Industrias Alimentarias. Universidad Privada Antenor Orrego. Trujillo Perú. Recuperado http://repositorio.upao. edu.pe/handle/upaorep/196

Braverman, J. (1990). Introducción a la Bioquímica de los Alimentos. México: El Manual Moderno, S.A. de C.V.

Casp, A. (2015). Tecnología de los Alimentos de Origen Vegetal. Volumen 2. España: Síntesis.

Cheftel, J., Cheftel, H., y Besancon, P. (1999). Introducción a la Bioquímica y Tecnología de los Alimentos. Volumen II. España: Acribia.
Escudero, J. (2008) Estudio e Investigación de la fruta de tamarindo (Tamarindus indica L.) y propuesta gastronómica. Tesis para Título profesional. de Administrador Gastronómico. Universidad Equinoccial. Quito - Ecuador. Disponible en https:// repositorio.ute.edu.ec

FAO y Comisión del Codex Alimentarius (1992). Zumos (jugos) de fruta y productos afınes, 2 edición. Roma: FAO, 1992. 121 p. 6

FAO/ Latin Foods (1997). Tabla de Composición de Alimentos de América Latina. Roma, Italia.

Hasan, S.K., y ljaz, S. (1972). Tamarind review. Sci. Ind. (Karachi).

Hernández, M. (2014). Desarrollo de cuatro formulaciones de helados a base de agua con bajo contenido de azúcar y enriquecido con vitamina C. Tesis para el Título profesional de Licenciado en Nutrición. Universidad Rafael Landivar. Guatemala. Recuperado de http://biblio3.url.edu.gt/ Tesario/2014/09/15/Hernandez-Maria.pdf

Hernández, F. (2016). Establecimiento de Cultivo "in vitro" de Tamarindus indica L. para la obtención de antioxidantes. Tesis para el Título profesional de Químico en Alimentos. Universidad Autónoma de México. Toluca. Recuperado de http://ri.uaemex.mx/bitstream/ handle/20.500.11799/65363/TESIS\%20 Tamarindo\%20completa.pdf?sequence $=3$

Kimball, D. (1999). Procesado de Cítricos. España: Acribia.

Lewis Y. J, Neelakatan, S., y Bhatia D.S. (1961). Organic acid metabolism in tamarind. 
Ordoñez, J. (1998). Tecnología de los Alimentos. Volumen I. Componentes de los Alimentos y Procesos. España: Síntesis.

Resolución Ministerial 591-2008/MINSA. Norma sanitaria que establece los criterios microbiológicos de calidad sanitaria e inocuidad para los alimentos y bebidas de consume humano.

Singh, R., y Heldman, D. (1997). Introducción a la Ingeniería de los Alimentos. España: Acribia.

Sosa Ingredients. Texturizantes y nuevas Tecnologías de los Sabores. Disponible de https://www.sosa.cat/catalogues/ texturizantes_nuevas_tecnologias_sabores. pdf. Soluciones Prácticas. Lima, Perú disponible en www.solucionespracticas. org.pe

Valdivia, J. (2017). Cambios Físico Químicos, Sensoriales y Nutricionales, debido a la evaporación de la Leche Fresca Entera. Tesis para Título profesional. UNALM. Lima, Perú. Recuperado de http:// repositorio.lamolina.edu.pe/bitstream/ handle/UNALM/3101/valdivia-calixtojorge-andres. pdf? sequence $=3 \&$ isAllowed $=y$ 